\title{
CAPILLARY FLOWS WITH FORMING INTERFACES
}

\section{Yulii D. Shikhmurzaev}

Professor of Applied Mathematics

University of Birmingham, UK

21 Chapman \& Hall/CRC Boca Raton London New York 


\section{Contents}

Preface $\quad$ ix

List of figures xiii

List of tables $\quad$ xvii

List of symbols $\quad$ xix

1 Introduction 1

1.1 Free-surface flows in nature and industry . . . . . . . . . . . 1

1.2 Scope of the book . . . . . . . . . . . . . 5

2 Fundamentals of fluid mechanics 9

2.1 Main concepts . . . . . . . . . . . . . . . . . 99 9

2.1.1 Physical properties of liquids and gases . . . . . . . 9

2.1.2 The continuum approximation: density and velocity . 11

2.1.3 The continuum approximation: forces and stresses . . 15

2.1.4 Eulerian and Lagrangian description of motion . . . . 20

2.2 Governing equations ................. 24

2.2.1 Conservation of mass . . . . . . . . . . . . . . 25

2.2.2 Equation of motion. Stress tensor . . . . . . . . . 28

2.2.3 Conservation of angular momentum. Symmetry of stress tensor ..................... 32

2.2.4 Ideal fluid . . . . . . . . . . . . . . . . . . 34

2.2.5 Elements of theory of deformation . . . . . . . . . 36

2.2.6 Viscous incompressible liquid . . . . . . . . . . . . . . . 41

2.3 Elements of thermodynamics . . . . . . . . . . . . . . . 52

2.3.1 General concepts . . . . . . . . . . . . . . . 52

2.3.2 Application to fluid .............. . . 55

2.4 Classical boundary conditions . . . . . . . . . . . . . 64

2.4.1 Boundaries and boundary conditions . . . . . . . . 64

2.4.2 Kinematic and dynamic conditions on liquid-fluid interfaces .................. 66

2.4.3 Conditions on solid boundaries . . . . . . . . . . 75

2.4.4 Conditions at three-phase contact lines . . . . . . . . . 79

2.5 Physically meaningful solutions and paradoxes of modelling . 87

2.5.1 'Standard model' ................ 87 
2.5.2 Analysis of results . . . . . . . . . . . . . . . 90

2.5.3 Paradoxes of modelling $\ldots \ldots \ldots$

3 Moving contact lines: An overview $\quad 97$

3.1 Essence of the problem . . . . . . . . . . . . . . 97

3.1.1 Spreading of liquids on solids . . . . . . . . . . . 97

3.1 .2 Liquid-liquid displacement . . . . . . . . . . . . . . . . 102

3.1 .3 Summary . . . . . . . . . . . . . . . . . 105

3.2 Experimental observations . . . . . . . . . . . . 105

3.2.1 Preliminary remarks . . . . . . . . . . . . . . 105

3.2 .2 Flow kinematics . . . . . . . . . . . . . . . . . 108

3.2 .3 Dynamic contact angle . . . . . . . . . . . . . . 117

3.2.4 Summary of experiments . . . . . . . . . . . . . 142

3.3 Molecular dynamics simulations . . . . . . . . . . . . 143

3.4 Review of theories . . . . . . . . . . . . . . . . 145

3.4.1 Slip models . . . . . . . . . . . . . . . . . . 146

3.4.2 Models developed for dynamic wetting by thin films . 161

3.4.3 Diffuse-interface models . . . . . . . . . . . . . 167

3.4 .4 Other models . . . . . . . . . . . . . . . . . . 171

3.4 .5 Summary . . . . . . . . . . . . . . . . . 173

3.5 The key to the moving contact-line problem . . . . . . 173

4 Boundary conditions on forming interfaces 177

4.1 Modelling of interfaces . . . . . . . . . . . . . . 177

4.2 Conservation laws . . . . . . . . . . . . . . . . 185

4.3 Liquid-gas and liquid-solid interfaces . . . . . . . . 190

4.3 .1 Dissipative mechanisms . . . . . . . . . . . . . 192

4.3.2 Estimates and further simplifications . . . . . . . 195

4.3.3 Surface equation of state . . . . . . . . . . . . 198

4.3.4 The simplest (irreducible) model . . . . . . . . . . . 201

4.3.5 Conditions at contact lines . . . . . . . . . . 206

4.4 Liquid-liquid interfaces . . . . . . . . . . . . . . . . 211

4.4.1 Conditions at contact lines . . . . . . . . . . . 214

4.5 Summary . . . . . . . . . . . . . . . . . . 217

4.6 Open questions and possible generalizations f . . . . . 218

5 Moving contact lines: Mathematical description 221

5.1 Flow in the immediate vicinity of a moving contact line . . . 221

5.1.1 Problem formulation . . . . . . . . . . . . . . 221

5.1 .2 Asymptotics as $r \rightarrow 0 \ldots \ldots \ldots \ldots . \ldots 224$

5.1.3 Implications for numerical computations . . . . . . . . 228

5.1.4 Qualitative illustration of the asymptotics . . . . . . 229

5.1.5 Summary of the near-field asymptotic analysis . . . . 231

5.2 Dynamic wetting at small capillary numbers . . . . . . . 232

5.2 .1 Dynamic contact angle . . . . . . . . . . . 235 
5.2.2 Forces near the moving contact line . . . . . . . . 239

5.2.3 Flow-induced Marangoni effect . . . . . . . . . . . . . 241

5.3 De-wetting and re-wetting . . . . . . . . . . . . 242

5.3.1 Liquid spreading over a pre-wet surface . . . . . . . . 243

5.3.2 Receding contact lines . . . . . . . . . . . . . . 245

5.4 Comparison with experiments and some estimates . . . . 247

5.5 Examples: flows in a quasi-static regime . . . . . . . . . . 254

5.5.1 Spreading of drops on solid substrates . . . . . . . 255

5.5.2 Bubble growth on a plate . . . . . . . . . . . 259

5.6 Dynamic wetting at finite capillary numbers . . . . . . . 262

5.6 .1 Problem formulation . . . . . . . . . . . . 262

5.6.2 The effect of the flow field/geometry on dynamic contact angle . . . . . . . . . . . . . . 264 264

5.6 .3 Flow field . . . . . . . . . . . . . . . . . 274

5.7 Liquid-liquid displacement . . . . . . . . . . . . . . 276

5.8 Summary and outstanding modelling issues . . . . . . . . 279

5.8.1 Dynamic wetting of imperfect substrates . . . . . . . 280

6 Cusps, corners and coalescence of drops $\quad 287$

6.1 Singularities of free-surface curvature in experiments . . . . 287

6.2 Conventional modelling . . . . . . . . . . . . . 290

6.2.1 Solution with a presumed singularity . . . . . . . . 290

6.2.2 Solution with an emerging singularity . . . . . . . . 294

6.2 .3 The paradox of cusping . . . . . . . . . . . . 299

6.3 'Missing' physics . . . . . . . . . . . . . . . . . . 300

6.3.1 Essential factors and additional influences . . . . . . 300

6.3.2 Singularity of curvature as a contact line in the interface disappearance process . . . . . . . . . . . . . 301

6.4 Singularity-free solution: cusp or corner? . . . . . . . . . . 304

6.4.1 Problem formulation . . . . . . . . . . . . . . 304

6.4.2 Near-field asymptotics . . . . . . . . . . . . . . . 306

6.4.3 Numerical and experimental implications . . . . . . 313

6.4 .4 Summary . . . . . . . . . . . . . . . . . . . . 314

6.5 Coalescence of drops . . . . . . . . . . . . . . . . . . . . 315

6.5.1 Coalescence in the standard model . . . . . . . . 317

6.5.2 Local self-similarity and the origin of the singularity . 324

6.5.3 Singularity-free description . . . . . . . . . . . 327

6.5.4 Computational and experimental aspects . . . . . 337

6.5.5 Summary . . . . . . . . . . . . . . . . 338

$\begin{array}{lll}7 & \text { Breakup of jets and rupture of films } & 341\end{array}$

7.1 Background . . . . . . . . . . . . . . 341

7.2 Drop formation: emerging singularity . . . . . . . . . 343

7.2 .1 Slender-jet approximation . . . . . . . . . . . . . . . . 343

7.2 .2 Similarity solutions . . . . . . . . . . . . . 347 
7.2 .3 Numerical results . . . . . . . . . . . . . . . . 350

7.3 Experiments on capillary pinch-off . . . . . . . . . . . 352

7.4 'Missing' physics and its qualitative verification . . . . . 360

7.5 Axisymmetric capillary pinch-off: singularity-free solution . . 364

7.5.1 Slender-jet approximation . . . . . . . . . . 365

7.5.2 Further simplifications: estimates for $Q_{1}$ and $\bar{\beta} \ldots 367$

7.5.3 Flow regimes . . . . . . . . . . . . . . 369

7.6 Pinch-off from a molecular viewpoint f . . . . . . . . 378

7.7 Rupture of films . . . . . . . . . . . . . . . . . . 381

7.7.1 Macroscopic mechanism . . . . . . . . . . . . 382

7.7 .2 Mesoscopic films . . . . . . . . . . . . . . . . . 389

7.8 Summary . . . . . . . . . . . . . . . . 395

A Elements of vector and tensor calculus $\quad 397$

B Equations of fluid mechanics in curvilinear coordinates $\quad 407$

C Complex representation of biharmonic functions 411

D Physical properties of some fluids $\quad 413$

$\begin{array}{ll}\text { References } & 415\end{array}$

Index of Authors $\quad 445$

Subject Index $\quad 453$ 